Juri Meda, Université de Macerata

\title{
"L'agonie de l'école " en Italie du sud dans les photographies de photojournalistes italiens, des années 40 aux années 50
}

Les classes dirigeantes ou les partis politiques ont souvent eu recours à la diffusion d'une image publique bien particulière des écoles rurales - toujours les écoles les plus pauvres et les plus désavantagées - pour documenter, selon leurs besoins en matière de propagande, l'état de modernité ou d'arriération du système scolaire national. À partir des reportages photo entrepris par des photojournalistes italiens et publiés dans les années 40 et 50 dans des magazines italiens aux orientations politiques diverses, cet article propose une analyse approfondie de la manière dont l'image publique de ces écoles a été manipulée et utilisée à des fins de propagande, par le biais de la diffusion de photographies dans des journaux et des magazines.

\section{Introduction}

Le reportage commandé par l'UNESCO sur la campagne contre l'analphabétisme en Calabre - un ensemble de photographies prises par David Seymour ${ }^{1}$ en 1950 dans les écoles de Roggiano Gravina, Cimino (un hameau près de San Marco Argentano), Saucci (un hameau près de Bagaladi) et San Nicola da Crissa - n'était pas le premier à souligner l'état déplorable de l'éducation publique dans le sud de l'Italie.

1 Le photographe n'a pas été choisi au hasard. En 1949, l'UNESCO avait publié un livre intitulé Children of Europe avec des photographies de David Seymour. Seymour, à la demande de l'UNICEF, s'était rendu en Autriche, en Grèce, en Italie, en Hongrie et en Pologne pour documenter les expériences vécues par des enfants après la guerre. Voir Samuel Boussian/Mathias Gardet/Martine Ruchat : Bringing Everyone to Trogen : UNESCO and the Promotion of an International Model of Children's Communities after World War II. In : Poul Duedahl (ed.) : A History of UNESCO : Global Actions and Impacts. Basingstoke : Palgrave Macmillan 2016, p. 99-115. Sur cette question, voir aussi Carole Naggar : Chim : Children of War. New York : Umbrage 2013 ; David Niget : Pieds nus dans les ruines : le regard de Chim sur les enfants de la guerre. In : Revue d'histoire de l'enfance irrégulière 15 (2013), p. 143-145.

2 Des photos de ce reportage ont été utilisées pour illustrer les articles suivants : Carlo Levi : L'Italie du sud mène la bataille contre l'analphabétisme. In : Le Courrier de l'UNESCO 5, nº 3 (1952), p. 3-5 ; Antonio Cortese : Youth Activities in Southern Italy. In : Fundamental and Adult Education 10, $\mathrm{n}^{\circ} 2$ (1958), p. 59-61. 
Après la Seconde Guerre mondiale - avec la levée du tabou sur les problèmes du système éducatif national qu'avait institué le régime fasciste ${ }^{3}$ - l'image publique de l'école s'est heureusement débarrassée de son uniformité factice. D'une part, les représentations de l'école ont trouvé leur inspiration dans le paradigme moderniste, reflétant la subordination de l'éducation à la " course à la modernité », qui allait devenir un aspect caractéristique des années de " boom économique »; d'autre part, la presse recommença à montrer les exemples les plus spectaculaires d'écoles délabrées en publiant des reportages photographiques aux objectifs politiques plus ou moins explicites.

Umberto Zanotti Bianco qui, dans les années 20, a mené des enquêtes photographiques sur l'état des écoles rurales dans le sud de l'Italie pour le compte de l'Associazione Nazionale per gli Interessi del Mezzogiorno d'Italia (Association nationale pour les intérêts de l'Italie du sud) a été le premier à lancer cette tendance. Son compte-rendu romanesque de son séjour en décembre 1928 à Africo, un petit village de montagne situé sur les pentes de l'Aspromonte, a été publié en plusieurs volets de mai à août 1946 dans la revue littéraire Il Ponte (fondée par Piero Calamandrei). ${ }^{4}$ Intitulé Tra la perduta gente (Africo) [ " Parmi les gens perdus (Africo) »], ce roman à épisodes dépeignait les conditions de vie misérables des habitants d'Africo, terrés dans leur taudis sans chauffage ni installations sanitaires, sous-alimentés et menacés par les maladies endémiques qui avaient fait monter en flèche le taux local de mortalité infantile. Son roman consacrait de longs passages à l'école :

Ce matin, avant de commencer mon enquête habituelle, j'ai choisi de visiter l'école. Favasuli m'a accompagné à la rencontre de la gentille petite institutrice qui enseigne aux classes combinées de seconde et troisième primaire. Elle a seulement vingt-six élèves inscrits, mais en fait il n'y en a qu'une vingtaine qui assistent aux cours. Étant donné qu'il est impossible de donner cours aux enfants dans la baraque délabrée, sans plancher ni fenêtre, où j'ai vu deux mules attachées, la malheureuse enseigne dans sa chambre à coucher, pratiquement dans le noir. « Il n’y a que quatre élèves qui ont payé leur bulletin scolaire », m’a-t-elle dit, désolée. " Je devrais renvoyer les autres... mais comment le pourrais-je ? La misère est vraiment répandue, comme vous l'aurez constaté. Je n'ai pas le cœur à le faire... L'autre jour, j’ai renvoyé un enfant à la maison parce qu'il n'avait pas de porte-plume : j'espérais qu'il irait en acheter un. Il n'est pas

3 Sur la censure stricte imposée par le régime fasciste à des fins de propagande sur l'image publique des écoles rurales dans le sud de l'Italie, voir Juri Meda : "Invisible Schools » : The Public Image of Rural Schools in Southern Italy in Photographic Inquiries and Photo-Reportages (19251955). In : Historia y Memoria de la Educación 8 (2018), p. 347-396, consulté le 5 mai 2019, doi : 10.5944/hme.8.2018.19204. Cet article faisait partie d’un numéro spécial intitulé « Fotografía, propaganda y educación » (Photographie, propagande et éducation), publié par Francesca Comas Rubi et María del Mar Pozo Andrés.

4 Umberto Zanotti Bianco : Tra la perduta gente (Africo) - I. In : Il Ponte (mai 1946), p. 405-414 ; Umberto Zanotti Bianco : Tra la perduta gente (Africo) - II. In : Il Ponte (juin 1946), p. 509-519; Umberto Zanotti Bianco : Tra la perduta gente (Africo) - III. In : Il Ponte (juillet-août 1946), p. 642648. 
revenu depuis. Seulement quatre enfants ont payé les cinq lires pour le manuel. Je l'ai commandé il y a quelque temps... mais rien n'est encore arrivé. Vous voyez... nous n'avons ni cahiers d'exercices ni plumes pour les enfants pauvres. Les cahiers d'exercices coûtent 25 centimes chacun, et les plumes coûtent trois lires la paire... qui a cet argent ? Je paierais volontiers, mais j'ai à peine de quoi subvenir à mes besoins. » L'institutrice de première année, qu'un passant avait appelée, a jeté un coup d'œil depuis le seuil de porte. Elle est exactement dans la même situation. Elle a quinze élèves inscrits, mais aucun n'a payé le bulletin scolaire. Elle a rassemblé dans la même classe ses élèves de première année et le seul élève de quatrième année primaire. J'ai noté tout ce dont ils avaient besoin et je vais le leur envoyer de Reggio, mais je sens monter dans mon cœur un sentiment de révolte. Ces pauvres malheureux ont-ils tort de ne voir l'État que sous les traits du fisc ou du garde forestier? Depuis vingt ans, ces gens vivent dans des conditions si dégradantes que quiconque ayant un minimum de foi dans l'humanité en rougirait de honte. ${ }^{5}$

Le récit largement salué de Zanotti Bianco avait le mérite de centrer le débat public sur la questione meridionale ${ }^{6}$ trop longtemps ignorée par le régime fasciste, présentant sa résolution comme une condition préalable nécessaire à la croissance sociale et économique d'un pays récemment sorti d'une guerre dévastatrice. L'état déplorable de l'éducation dans les régions méridionales était ainsi replacé une fois encore au cœur même de cette question.

Zanotti Bianco a joué un rôle crucial pour alerter l'opinion publique mais son intervention n'aurait pas suffi à attirer l'attention des médias sur la situation inquié-

5 « Stamane, prima di iniziare la mia solita inchiesta, ho voluto visitare la scuola. Favasuli mi ha accompagnato dalla mite maestrina che dirige la seconda e terza elementare riunite. Non ha che ventisei iscritti di cui solo una ventina frequentano effettivamente le classi. Poiché non è possibile far scuola nella sconnessa baracca senza pavimento, senza finestre, ove ho visto stavano rinchiuse due mule, la poveretta insegna nella sua camera da letto, quasi al buio. « Solo quattro alunni - mi dice con voce sconsolata - hanno pagato le pagelle. Dovrei mandar via gli altri... ma come fare ? La miseria, come ha potuto constatare, è veramente generale. Non ne ho cuore... L'altro giorno ho rinviato un bimbo perché non aveva la penna, sperando che andasse a comperarla : non è più tornato. Quattro bimbi soltanto hanno versato le cinque lire per il libro di testo... È da tempo che l'ho commissionato... nulla è ancora giunto. Vede... manchiamo completamente di quaderni, di pennini per i poveri : i quaderni costano 25 centesimi, i pennini vanno a tre soldi due... chi ce li mette ? Lo farei volentieri, ma ho appena quanto mi basta per tirare avanti. > La maestra di prima, che, avvisata da qualche passante, s'è affacciata all'uscio, è nelle identiche condizioni. Ha quindici iscritti, nessuno ha però voluto pagare la pagella : ha unito agli alunni di prima classe anche l'unico frequentante della quarta elementare. Ho preso nota di tutto il fabbisogno, che manderò da Reggio, ma un moto di ribellione mi agita il cuore. Hanno torto questi disgraziati a non vedere nello Stato che il fisco sotto veste d'esattore o di milite forestale ? Da più di vent'anni questa popolazione vive in condizioni così degradanti da far arrossire di vergogna chi ha un po' di fierezza umana. » Zanotti Bianco : Tra la perduta gente (Africo) - II. In : Il Ponte (juin 1946), p. 513-14. Sauf indication contraire, toutes les traductions sont de l'auteur.

6 Dans l'historiographie italienne, ce terme est utilisé pour décrire le débat autour des raisons qui devaient conduire et, avec le temps, aggraver le sous-développement social et économique du Sud à la suite de l'unification de l'Italie. 
tante des écoles dans les régions centrales du Sud sans la présence de trois autres facteurs : 1) le développement des agences photo spécialisées dans la photo d'actualité au lendemain de la guerre qui à leur tour ont créé le métier de photojournaliste et utilisé de plus en plus couramment l'appareil photo comme outil d'enquête sociale ; 2) la soif de vérité qui gagnait un pays habitué depuis vingt ans à une représentation idyllique de la réalité, nourrie par la propagande du régime fasciste, une réalité certes rassurante, mais en même temps monotone et déformée ; et 3) l'extraordinaire succès national des magazines d'information richement illustrés traitant de l'actualité, de modes de vie et de reportages thématiques et dont le tirage a atteint le chiffre de 4,5 millions d'exemplaires par semaine de 1947 à 1952. ${ }^{7}$ Comme l'ont fait remarquer Uliano Lucas et Tatiana Agliani :

Cela a commencé avec des photos de la reconstruction, avec des images de la vie reprenant son cours dans les grandes villes, de la reconstruction et de la réouverture des usines, et $d u$ retard du Sud, longtemps passé sous silence ; des reportages sur les évènements les plus importants de l'actualité, les crimes et les histoires d'amour que l'Italie de Mussolini avait dissimulés aux regards de la population, et qu'un public assoiffé de savoir, et avide de connaître ce qui se passait vraiment dans le pays, exigeait de cette nouvelle presse, des quotidiens du matin et du soir, et des hebdomadaires qui avaient commencé à paraître et à se multiplier en l'espace de quelques années jusqu'à atteindre, au début des années cinquante, le chiffre le plus élevé de toute l'Europe proportionnellement à la population. ${ }^{8}$

Juste après la guerre, cette combinaison complexe de causes simultanées a entraîné un essor des reportages photographiques, symboles mêmes d'une liberté de la presse que le pays retrouvait tout juste. Influencée par le courant néoréaliste alors très en vogue dans l'industrie cinématographique, la presse a cherché à dépeindre la réalité de façon très réaliste, sans filtre ni interprétation, et en mettant notamment l'accent sur les conditions de vie des classes les plus pauvres. Les écoles du Sud, dont les problèmes séculaires étaient encore une fois sous les feux de l'actualité, constituaient le sujet idéal. ${ }^{9}$

7 Voir Silvia Pizzetti : I rotocalchi e la storia. Rome : Bulzoni 1982, p. 22.

8 « Inizia con le foto della ricostruzione, con le immagini della vita che riprende nelle grandi città, del ricostituirsi delle fabbriche, dell'arretratezza a lungo taciuta del Meridione, con la documentazione dei grandi fatti d'attualità, di cronaca nera e rosa che l'Italia mussoliniana aveva nascosto allo sguardo degli italiani e che ora una popolazione assetata di sapere, desiderosa di vedere la vera realtà del Paese, richiede alla nascente stampa, ai quotidiani del mattino e del pomeriggio, ai settimanali che si vanno moltiplicando in pochi anni fino a raggiungere, nei primi anni Cinquanta, il numero maggiore in Europa in proporzione agli abitanti. » Uliano Lucas/Tatiana Agliani : L'immagine fotografica 1945-2000. In : Uliano Lucas (éd.) : Storia d'Italia. Annali 20. L'immagine fotografica 1945-2000. Turin : Einaudi 2004, p. 5, voir surtout le passage intitulé « La scoperta della fotografia realista nell'Italia del dopoguerra », p. 4-19.

9 Les principales revues à vocation pédagogique du Sud ont commencé à mener leurs premières enquêtes sur ce thème à ce moment précis ; voir Vincenzo Muro : L'analfabetismo in Italia e la necessità di combatterlo. In : Scuola nostra : rivista quindicinale della scuola elementare del Mezzo- 


\section{Le reportage photo de Tino Petrelli sur Africo (1948)}

Ce n'est donc pas une simple coïncidence qui a incité l'hebdomadaire milanais L'Europeo à publier, en mars 1948, une série de photographies du village calabrais d'Africo où le temps semblait s'être arrêté depuis $1928 .{ }^{10}$ Ces photographies, d'une extraordinaire éloquence visuelle, ont été prises par le photographe Tino Petrelli, ${ }^{11}$ un correspondant de l'agence Publifoto, pour illustrer un reportage complexe sur les conditions de vie dans le Sud, mis sur pied par Arrigo Benedetti et mené par le journaliste Tommaso Besozzi pour le compte de L'Europeo. Les photographies de Petrelli ont été utilisées pour illustrer deux articles : deux clichés pris à l'intérieur de l'école d'Africo accompagnaient un article sur la mystique calabraise Fortunata Evolo, ${ }^{12}$ tandis que deux autres, qui témoignaient des conditions de vie déplorables dans les maisons de village où hommes et animaux vivaient côte à côte, ont été publiés dans un article sur le village d'Africo lui-même. ${ }^{13}$ Les deux photographies prises à l'école ont été magistralement décrites par l'anthropologue Vito Teti :

$\overline{\text { giorno }}^{\mathrm{er}}$ mai 1948), p. $3-4$; 3.000 alunni in 16 aule. In : Il Mezzogiorno : rassegna della vita e dei problemi del Sud (10 décembre 1948), p. 22 ; Nicolò Piccinni : Il problema della scuola nel Mezzogiorno. In : Scuola nostra (5 janvier 1949), p. 1-2.

10 L'hebdomadaire L'Europeo - fondé par Arrigo Benedetti en 1945 et publié par Editoriale Domus jusqu'en 1953, date à laquelle il a été vendu à Rizzoli - est devenu célèbre pour avoir adopté un design novateur basé sur l'utilisation du même format que les quotidiens, lui permettant ainsi d'accueillir des solutions graphiques innovantes et d'avoir une mise en page extrêmement flexible.

11 Valentino (Tino) Petrelli (1922-2001) a commencé à travailler pour l'agence de photographie Publifoto de Vincenzo Carrese en 1937. Il était chargé de développer des négatifs et d'effectuer les tirages des photographies. Carrese a été la première personne dans la période d'après-guerre à avoir compris le potentiel du nouveau marché éditorial. Il a transformé son agence qui deviendrait bientôt la plus importante agence photographique de presse, fournissant les plus grands titres d'Italie. Engagé comme photographe professionnel, Petrelli est devenu l'un des assistants les plus proches de Carrese, et a réalisé de nombreux reportages photographiques réussis, par exemple sur Piazzale Loreto (1945), Africo (1948) et les inondations de Polesine (1951). Pour plus de renseignements, voir Italo Zannier (éd.) : Tino Petrelli : fotogiornalismo in Italia. Pordenone : Edizioni Concordia Sette 1980.

12 Tommaso Besozzi : L'errore del vescovo di Mileto : Da dieci anni la scienza studia Natuzza dei Morti ma per i calabresi essa è la donna del miracolo. In : L'Europeo (14 mars 1948), p. 8.

13 Tommaso Besozzi : Troppo strette le strade per l'ombrello aperto. Il più disperato paese della Calabria. In : L'Europeo (21 mars 1948), p. 6. Entre autres choses, l'article disait que « Ad Africo [...] non c'è acqua né luce elettrica ; non ci sono botteghe, né locande; la gente mangia un pane color cioccolata, fatto di farina di lenticchie selvatiche ; le abitazioni, tolte pochissime, sono di un locale solo e là vivono assieme uomini e bestie. Ad Africo esistono solo tre case provviste di latrina e ci sono solo tre persone che posseggono un ombrello. Ma, essendo le strade del paese troppo strette perché ci si possa aprire un ombrello, se ne debbono servire unicamente quando vanno a Bova o a Motticelle. » (« À Africo [...] il n’y a ni eau ni électricité ; il n’y a ni magasins ni auberges ; les gens 
Six fillettes sont assises à des bureaux en bois, quatre faisant face à la caméra et quatre vues de côté. Elles sont toutes concentrées et penchées sur leurs manuels, presque comme si elles y cherchaient quelque chose de caché, de profond. Aucune ne regarde l'objectif. Elles semblent toutes fascinées par les chiffres et les mots ; une fillette au deuxième rang paraît examiner quelque chose qu'elle ne peut pas tout à fait comprendre, tandis qu'une autre porte une coiffe en laine d'où dépassent ses cheveux. Les lames de plancher disloquées nous font comprendre que nous nous trouvons dans un environnement vieux et misérable. Une carte déchirée de la Calabre se trouve sur le mur du fond à gauche de l'image. Deux fillettes pieds nus tentent de se réchauffer les pieds à deux braseros de métal contenant des cendres et du charbon de bois, placés au pied des bureaux. ${ }^{14}$

mangent du pain de couleur chocolat fait à base de farine de lentille sauvage ; les maisons, à l'exception de très peu d'entre elles, n'ont qu'une seule pièce partagée par les hommes et les animaux. À Africo, il n’y a que trois maisons avec des toilettes et trois personnes seulement possèdent un parapluie. Mais étant donné que les rues du village sont trop étroites pour ouvrir un parapluie, elles ne peuvent les utiliser que lorsqu'elles se rendent à Bova ou à Motticelle. ") L'article mentionne Umberto Zanotti Bianco, qu'il appelle « Le grand protecteur d'Africo » ("grande protettore di Africo »), mais indique à tort qu'il « a produit un précieux rapport qui a provoqué un énorme retentissement » (« fece una relazione coraggiosa che sollevò molto scalpore ») et qui l'a conduit en prison ; il est vrai que Zanotti Bianco a été surveillé par la police fasciste à partir de 1928 - comme on peut le voir dans son dossier personnel, $\mathrm{n}^{\circ}$ 55044, qui se trouve maintenant au Casellario Politico Centrale (Bureau central des archives politiques) aux Archives centrales de l'État à Rome - mais il n'a été envoyé à l'isolement qu'en 1941.

14 « Tra i banchi di legno sono sedute sei bambine, quattro in posizione frontale rispetto al fotografo, due di fianco. Sono tutte intente e assorte sui loro sussidiari, quasi impegnate a cercare qualcosa di nascosto e di profondo. Nessuna guarda nell'obiettivo, tutte sembrano attratte dalle figure e dalla scrittura ; una bimba in seconda fila sembra guardare qualcosa di imprendibile. Un'altra ha sulla testa un copricapo di lana, che le lascia fuori i capelli. Le tavole divelte di legno fanno capire che siamo in un ambiente antico e precario. Appesa alla parete in fono a sinistra dell'inquadratura, la carta geografica strappata della Calabria. Due delle bambine hanno i piedi scalzi, che tentano di rifugiarsi nei due bracieri di lamiera ai lati del banco nei quali si scorgono la cenere e la carbonella. » Vito Teti : Il senso dei luoghi : memoria e storia dei paesi abbandonati. Rome : Donzelli 2004, p. 222. 


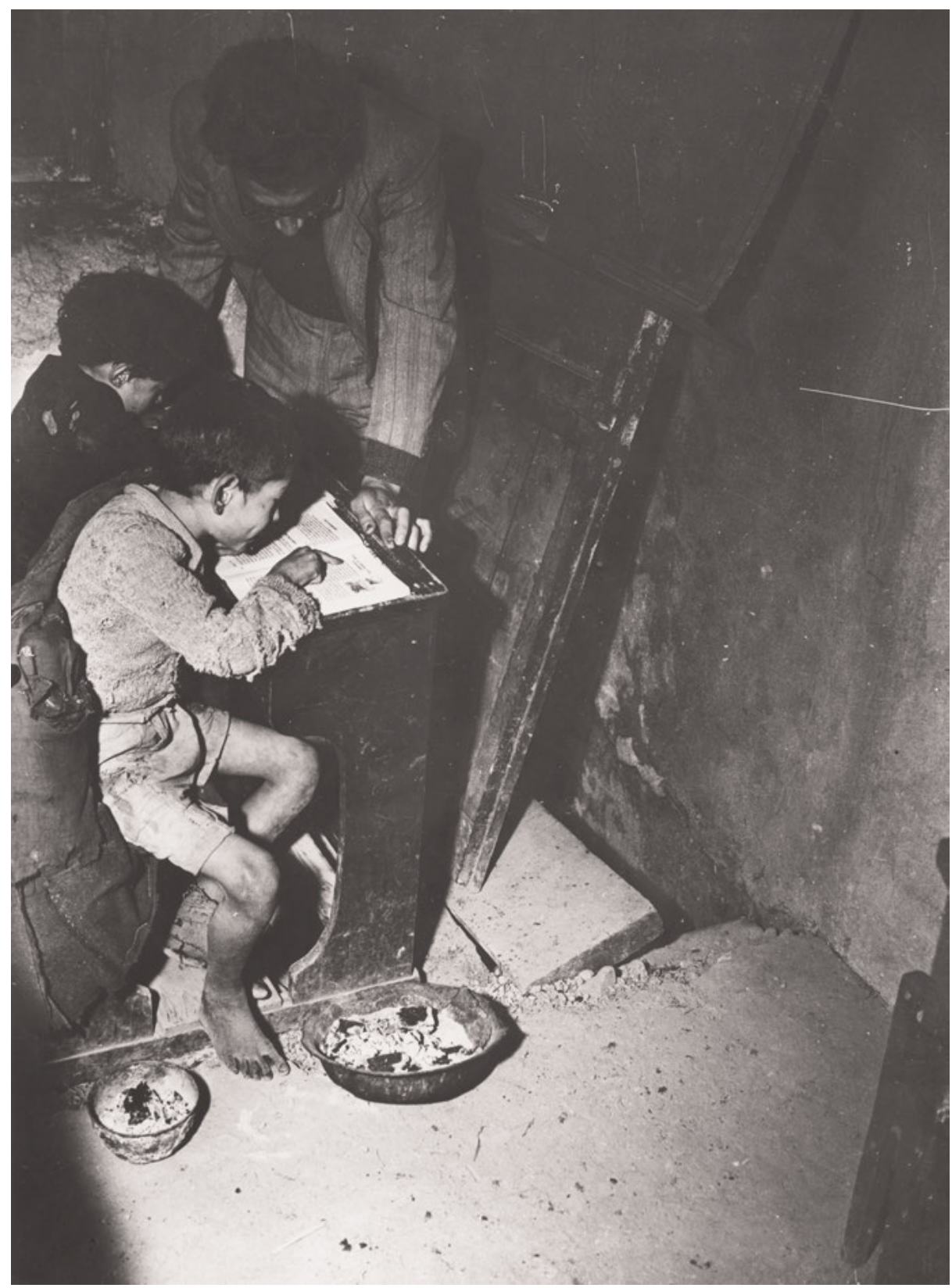

Fig. 3.1 Valentino Petrelli, Intérieur de l'école primaire à Africo (1948) @ Archivio Publifoto Intesa Sanpaolo - Région de Lombardie / Musée de la photographie contemporaine, Milan-Cinisello Balsamo. 


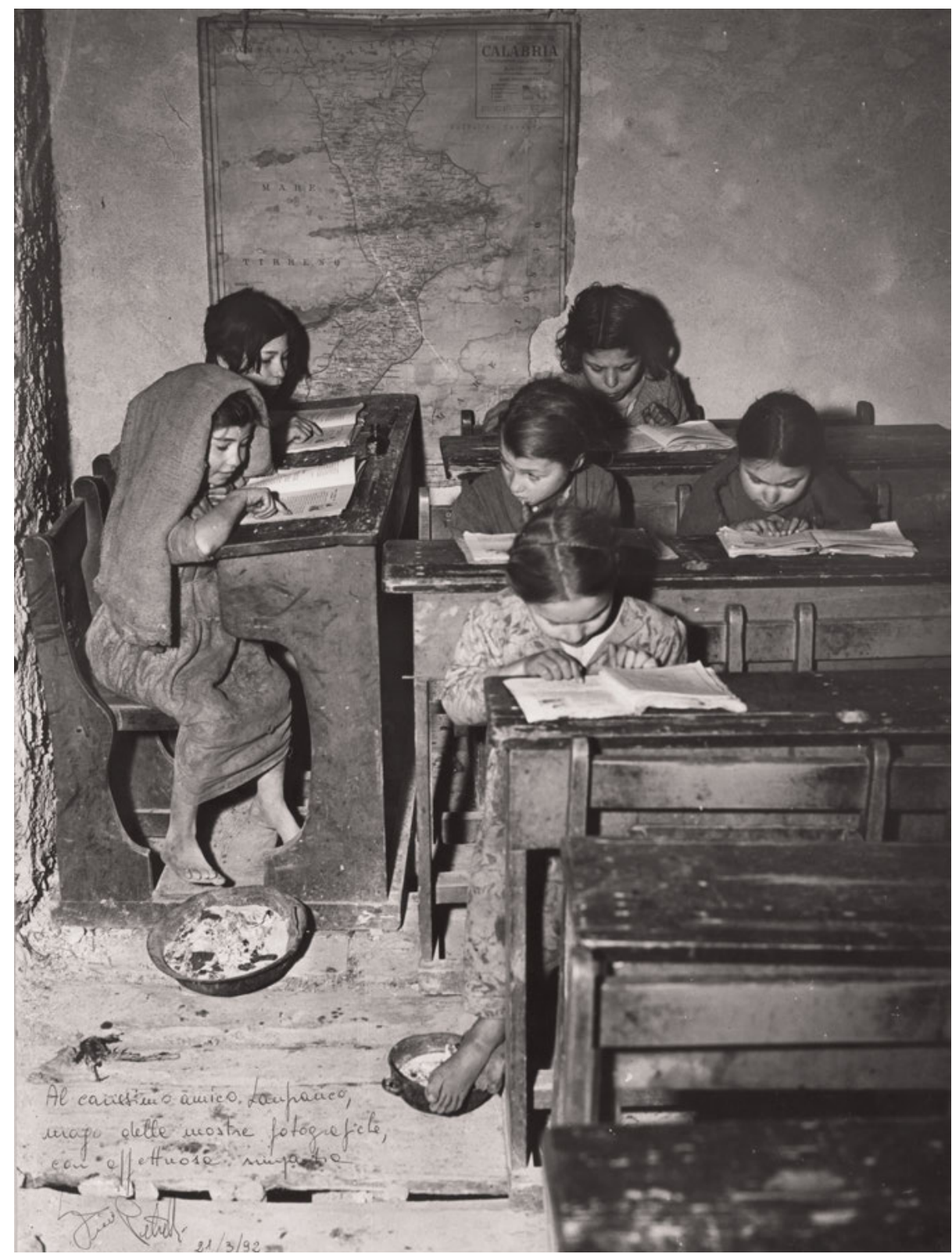

Fig. 3.2 Valentino Petrelli, Intérieur de l'école primaire à Africo (1948) @ Archivio Publifoto Intesa Sanpaolo - Région de Lombardie / Musée de la photographie contemporaine, Milan-Cinisello Balsamo.

Il est à noter ici que les photographies de l'état pitoyable de l'école à Africo n'ont pas été publiées, comme on pouvait s'y attendre, pour illustrer l'article sur le village 
lui-même, mais pour accompagner un article sur une femme qui a souvent accueilli chez elle des gens venus du monde entier à la recherche de nouvelles de leurs proches décédés ou d'informations sur leurs maladies, témoin vivant de la survie de superstitions et de croyances ancestrales dans la culture arriérée du sud de l'Italie. La combinaison de mots et d'images, qui n'était, encore une fois, pas une simple coïncidence, a implicitement amené le lecteur à se poser la question suivante : comment les peuples du Sud peuvent-ils rattraper le monde moderne si même leurs écoles, ces véritables phares de la civilisation, sont dans une état aussi épouvantable ? Par la force visuelle de ses images, Petrelli a réussi à rendre évidente la condition d'arriération généralisée du Sud en décrivant une école qui échoue non seulement de toutes les manières possibles à s'inscrire dans le paradigme esthétique des écoles en tant que facteurs de modernisation, mais qui révèle le grave sous-développement et la paralysie qui affligent la société du Sud dans son ensemble. Ses « scènes intemporelles » évoquent le passé, pas le présent ni l'avenir. Ce que nous voyons ne correspond pas à la date que nous avons intuitivement attribuée à l'image en fonction d'autres critères " objectifs " (le cadrage et la définition de l'image, la qualité de l'impression, etc.). C'est pourquoi nous sommes si souvent désorientés par ces photographies. Le reportage de Petrelli démontre son immense talent pour l'exploration ethnographique, ce qui est crucial pour interpréter correctement les scènes navrantes de la vie quotidienne qu'il a constatées à Africo. ${ }^{15}$

Cependant, ces photographies servent également un objectif différent qui ressort d'une analyse des légendes originales publiées dans L'Europeo. La légende de la figure 1 se lisait comme suit : «Il n'y a pas de fenêtre ; le chauffage consiste en ce que les étudiants peuvent apporter de chez eux - quelques braises dans une vieille bassine. Les problèmes du Sud sont légion, mais les écoles de la région les incarnent tous. Les problèmes n'ont pas été résolus par la dictature fasciste et ne seront résolus par aucune autre dictature, mais par l'intérêt qu'aura le pays pour cette question. ${ }^{16}$ La légende de la figure 2 semblait poursuivre ce fil conducteur : « Maintenant, à la veille des élections, même la pauvreté des Calabrais risque de devenir

15 Il convient de préciser ici que, durant ces mêmes années, de nombreux photographes ont documenté les expéditions scientifiques dans le Sud menées par des anthropologues italiens comme Arturo Zavattini et Franco Pinna dans le contexte de leur recherche dans les domaines du folklore et de l'ethno-musicologie. Sur les photographies ethnographiques, voir Diego Carpitella : Franco Pinna e la fotografia etnografica in Italia. In : Franco Pinna (éd.) : Viaggio nelle terre del silenzio : Fotografie di Franco Pinna. Milan : Idea Editions 1980, p. 4-11; Franceso Faeta (éd.) : Arturo Zavattini fotografo in Lucania. Milan : Federico Motta Editore 2003 ; Francesco Faeta : Fotografi e fotografie : Uno sguardo antropologico. Milan : FrancoAngeli 2006, surtout le chapitre intitulé « Il sonno sotto le stelle : Arturo Zavattini, Ernesto de Martino, un paese lontano », p. 113-139.

16 « Mancano le finestre, il riscaldamento è quello che gli scolari si procurano portandosi da casa qualche brace in un vecchio catino. Il problema del Mezzogiorno ha vari aspetti, ma quello delle scuole li assomma tutti. Non lo risolse la dittatura fascista, non lo potrà risolvere un'altra dittatura, ma l'interessamento del Paese. » Besozzi : L'errore del vescovo di Mileto, p. 8. 
un thème de campagne électorale. Les propagandistes montent depuis les plaines, cherchant à réveiller des hommes et des femmes dont le cerveau est affaibli par le besoin. $»^{17}$

Il ressort clairement de ces légendes que les reportages photographiques étaient utilisés comme une forme subtile de propagande par un hebdomadaire laïque et libéral dont les trois objectifs politiques fondamentaux étaient : 1) dénoncer la débâcle du régime fasciste qui, malgré ses nombreuses déclarations, n’avait pas réussi à desserrer la prise de l'analphabétisme qui tenait le Sud à la gorge ;2) condamner implicitement le cynisme des propagandistes communistes, prêts à spéculer sur la pauvreté de longue date de ces gens « dont le cerveau était affaibli par la pauvreté » afin de recueillir les voix dont ils avaient besoin pour gouverner (« l'autre dictature » incapable de résoudre le problème était la dictature prosoviétique qui, selon la rhétorique anticommuniste, entrerait au pouvoir si la gauche remportait les élections ${ }^{18}$; et 3) présenter de manière subliminale à l'opinion publique les conséquences possibles d'une suspension du plan Marshall qu'annoncerait le gouvernement américain en cas de victoire des communistes aux élections législatives du 18 avril 1948, quelques jours après la publication des photographies.

\section{Les « écoles impossibles » de Federico Patellani (1952)}

En juin 1952, le photographe Federico Patellani ${ }^{19}$ publiait dans l'hebdomadaire $E_{\text {poca }}{ }^{20}$ une autre campagne de photojournalisme qui enquêtait sur l'état des écoles

17 « Ora alla vigilia delle elezioni anche la miseria delle popolazioni calabresi rischia di diventare un motivo elettorale. Dalla pianura salgono i propagandisti che cercano di destare uomini e donne intontiti dal bisogno. » Ibid.

18 Le 20 janvier 1945, des habitants d'Africo attaquèrent le poste local des carabinieri, forçant les officiers présents à chercher refuge dans la cave et ne les libérant qu'après les avoir désarmés. À la suite de cet épisode, des antennes du Parti socialiste et du Parti communiste ainsi qu'un siège syndical de gauche ont été installés dans le village. Pour plus de détails sur cet épisode, voir Vito Teti : Il senso dei luoghi. Rome : Donzelli 2014, p. 225-226.

19 Federico Patellani (1911-1977), photographe, a commencé à travailler exclusivement pour l'hebdomadaire Tempo en 1946, renouvelant profondément la façon dont les lecteurs étaient informés grâce à ce qu'il appelait des " photo-textes ", qui consistaient en une couverture photographique complète avec des légendes qu'il écrivait lui-même, renversant totalement la relation hiérarchique entre texte et image. Il a cessé de travailler pour Tempo en 1952 et a fondé Pat Photo Pictures, où il travaillait en indépendant et bénéficiait de la coopération de nombreux photographes. Voir Federico Patellani : Federico Patellani: documenti e notizie raccolti in trent'anni di viaggio nel Sud. Milan : Editphoto 1977 ; Kitti Bolognesi/Giovanna Calvenzi (éds.) : Federico Patellani : fotografie e cinema 1943-1960. Prato : Archivio Fotografico Toscano - Région Toscane 2005 ; Kitti Bolognesi/Giovanna 
dans le Sud. À l'époque, Patellani avait participé à un important reportage intitulé «Italia Magica ${ }^{21}$ pour l'hebdomadaire Tempo, qui appartenait au même groupe d'édition. ${ }^{22}$ Comme cela avait été le cas pour le reportage de Petrelli, la combinaison des intérêts ethnographiques de l'auteur et de ses intentions documentaires a façonné la perspective à partir de laquelle le rapport examine la situation dans les écoles, sauf que dans le cas de Patellani, l'idée de l'exposé social est centrale et que ses images ne sont pas soumises à des fins politiques. Patellani écrivait dans l'article d'Epoca :

Il faut visiter la région pour comprendre la gravité du problème des écoles dans certaines régions du Sud, un problème que les rhétoriciens ont mis au placard depuis des décennies. Dans certains villages, les enfants doivent parcourir plus de dix kilomètres à pied pour se rendre à l'école. Et leurs écoles sont presque toujours situées dans des couloirs sans fenêtre, des anciennes écuries et étables ou des hangars à outils agricoles où la seule ouverture est la porte. Les photographies publiées ici décrivent ces scénarios incroyables mieux que tous les mots. Souvent, près de l'ancienne écurie, il y a une étable, et les mots de l'enseignant sont soulignés en permanence par le beuglement des vaches, voire le grognement des porcs. Dans un petit village de Calabre, j'ai vu une salle de classe par laquelle une vache et son veau devaient passer pour arriver au pâturage, car seule une cloison de planches séparait la salle de classe de l'étable. Quand le veau n'était pas dans le pré, il était particulièrement agité et bruyant, brisant les planches avec ses cornes et son museau, de sorte que la salle de classe

Calvenzi (éds.) : Federico Patellani : professione fotoreporter. Cinisello Balsamo : Museo della fotografia contemporanea - Silvana Editoriale 2015.

20 Federico Patellani : Le scuole impossibili : scantinati, antiche stalle, corridoi senza finestre sono diventati aule ; in qualche classe mancano i banchi, gli sgabelli e perfino il gesso per scrivere sulla lavagna. In : Epoca (28 juin 1952), p. 44-48. L'hebdomadaire illustré Epoca fondé par Alberto Mondadori en 1950 et publié jusqu'en 1997, se distingue par sa mise en pages unique, qui utilise abondamment le papier glacé et les reportages photographiques.

21 Le reportage «Italia Magica » commençait par l'article Le streghe di Benevento hanno paura dei fari (« Les sorcières de Benevento ont peur des phares »). In : Tempo (12 avril 1952), p. 18 : « Teтpo inizia con questo fototesto la pubblicazione di una serie di servizi che Federico Patellani ha realizzato andando a zonzo per l'Italia senza una meta precisa, ma ascoltando tutti i discorsi, interrogando la gente più semplice e troppo lontana dai beni che la civiltà offre ai popolo più fortunati ; alla ricerca di leggende, personaggi e situazioni che racchiudono ancora in sé un po' di quella magia che il progresso non è riuscito a estinguere. » ( Avec ce phototexte, Tempo lance la publication d'une série d'enquêtes que Federico Patellani a réalisées en voyageant en Italie sans but précis, mais en écoutant simplement ce que la population avait à dire, en interrogeant les gens les plus simples et les plus éloignés des avantages que la civilisation offre aux plus fortunés ; à la recherche de légendes, de personnages et de situations recélant encore un peu de cette magie que le progrès n'a pas réussi à éteindre. ) La série se terminait par l'article suivant : Si vince il gallo se si conosce la Traviata. In : Tempo (12 juillet 1952), p. 30-31.

22 Cet hebdomadaire illustré - fondé par Alberto Mondadori en 1939 et vendu à Aldo Palazzi en 1946 - s'inspire de l'hebdomadaire américain Life et voulait concurrencer Oggi, un hebdomadaire publié par son concurrent Rizzoli. Ce fut le premier magazine couleur italien à attribuer une importance égale aux journalistes et aux photographes : toutes les photographies étaient signées du nom du photographe, au même titre que les articles étaient signés du nom de l'auteur. 
contenait en réalité non seulement l'enseignant et ses élèves, mais l'enseignant, ses élèves et le veau. En hiver, d'énormes bouffées de vapeur sortaient de la gueule de l'animal et, franchement, cela n'aidait pas à purifier l'air. Pourtant, ni le veau, ni l'enseignant et ses élèves ne semblaient dérangés par la présence de l'autre. (Malheureusement, la scène a également eu pour témoin un journaliste britannique qui menait la même enquête que moi). ${ }^{23}$

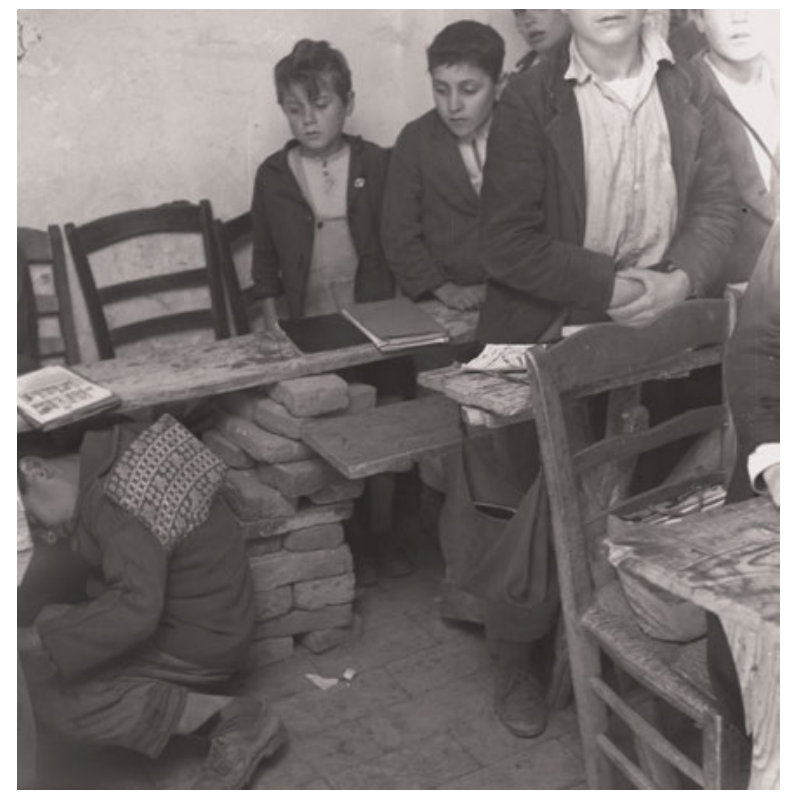

Fig. 3.3 Federico Patellani, Intérieur de l'ècole primaire de Jotta (1952) @ Studio Federico Patellani Région de Lombardie / Musée de la photographie contemporaine, Milan-Cinisello Balsamo.

23 « Bisogna venire da queste parti per intendere la gravità del problema scolastico in certe zone del Sud ; un problema tenuto nel dimenticatoio, per decenni, da tanti e tanti retori. In certi paesi i bambini devono percorrere, a piedi, oltre dieci chilometri per raggiungere la scuola. Questa, quasi sempre, è situata in un corridoio senza finestre, o in una antica stalla, o in un locale destinato agli attrezzi agricoli, senz'altra apertura che la porta. Le fotografie che qui pubblichiamo commentano meglio di qualsiasi parola quegli incredibili scenari. Spesso, vicino all'antica stalla, c'è una stalla ancora in efficienza e le parole dell'insegnante risultano perennemente commentate dai muggiti delle mucche e dai grugniti dei porci. In un paesino della Calabria m'è accaduto addirittura di vedere un'aula attraverso la quale doveva passare, per andare al pascolo, la mucca col vitellino. Infatti l'aula era divisa dalla stalla solo per mezzo di un assito. Quando non era al pascolo, il vitellino si dimostrava particolarmente inquieto, e faceva un gran fracasso tirando cornate e musate contro la paratia. Con questo metodo era riuscito a spostare due tavole per infilarci il muso. Così, in quell'aula, non c'erano soltanto gli scolari e il maestro : ma gli scolari, il maestro e un vitellino. D'inverno, dalla bocca della bestia uscivano ondate di vapore e non si può dire che l'aria ne godesse. Ma né l'uno né gli altri sembravano disturbati dalla reciproca presenza. (Queste cose, purtroppo, furono viste e annotate anche da un giornalista inglese che faceva la mia medesima inchiesta). » Patellani : Le scuole impossibili, p. 45. 


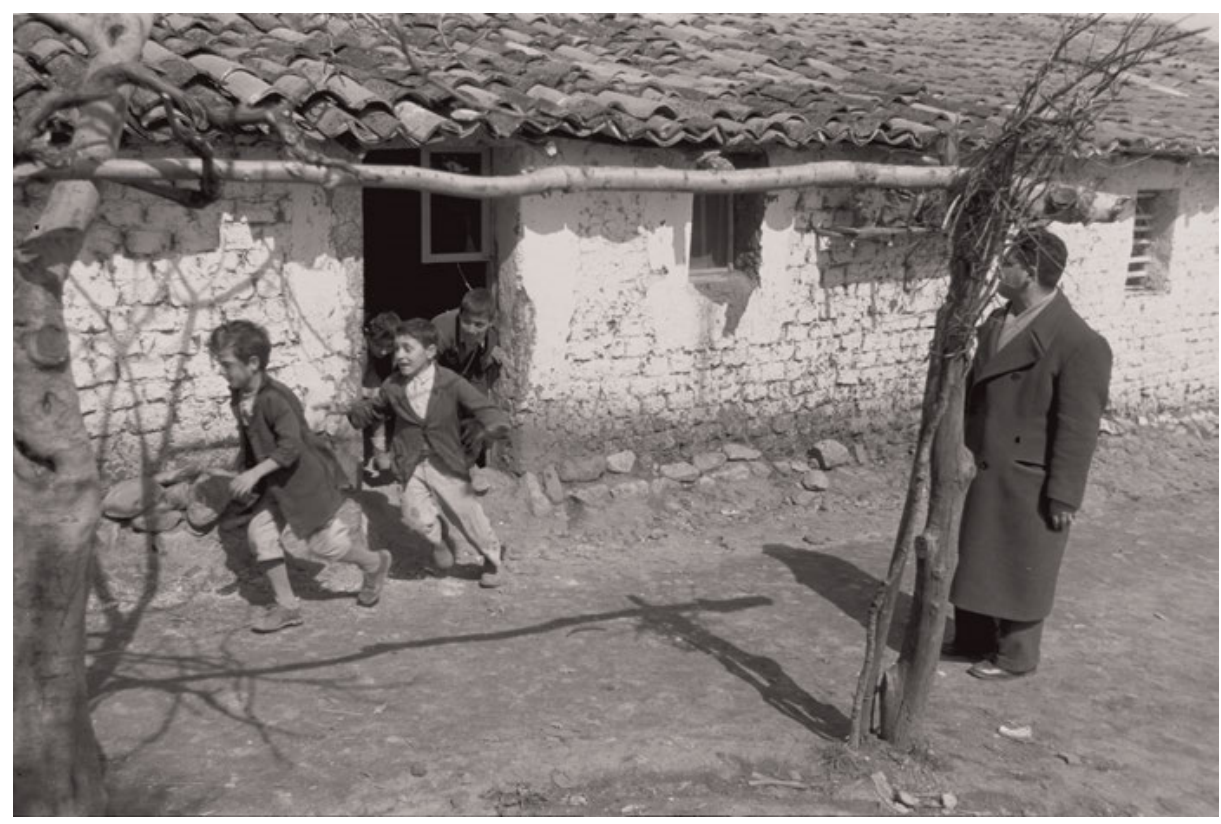

Fig. 3.4 Federico Patellani, Extérieur de l'école primaire de Jotta (1952) @ Studio Federico Patellani Région de Lombardie / Musée de la photographie contemporaine, Milan-Cinisello Balsamo.

Patellani a ensuite décrit la situation qu'il avait rencontrée dans les écoles rurales de Jotta et Ghiandaro, deux petits faubourgs de San Marco Argentano, dans la province de Cosenza : « À Jotta, les bureaux étaient tellement hauts que les élèves, même en grimpant sur leurs chaises dépaillées, pouvaient à peine poser le menton dessus ", ${ }^{24}$ alors qu'à Ghiandaro, parce qu'ils avaient été donnés par une école maternelle du nord de l'Italie, « les bureaux dans la salle de classe de deuxième année primaire étaient si petits que les enfants, arrivaient à peine à s’y faufiler $»{ }^{25} \mathrm{Il}$ a conclu:

Très peu de choses ont été faites pour les enfants qui vont à l'école dans certaines régions du Sud. C'est avec un brin de remords que nous pensons aux nombreux édifices publics pompeux des grandes villes (les gares ferroviaires avec leurs colonnes assyriennes et babyloniennes, les villes modèles plaquées de marbre et les bureaux de poste qui ressemblent à des mausolées) lorsque nous voyons les salles de classe de Jotta, Ghiandaro, Matera et d'innombrables autres villages de Calabre, de Basilicate et des Pouilles ; ces villages où les enfants grandissent assis à des bureaux impossibles, redoublant sans cesse l'année scolaire jusqu'à ce que vienne l'heure

24 «I banchi erano così giganteschi che i bambini, arrampicati sopra le seggiole spagliate, potevano a malapenaarrivarci col mento. » Ibid., p. 45.

25 « Nelle aule di seconda elementare i banchi sono talmente minuscoli che i bambini quasi non c'entrano e devono schiacciarvisi dentro. » Ibid., p. 46. 
d'aller travailler et de reléguer l'école à leurs souvenirs : un souvenir de paille et de plâtre émietté tombant du plafond. [...] Bref, ce voyage dans la région des écoles improbables m’a amené à conclure que dans certaines régions, tout est à faire en partant de zéro [...] Sans exagération, on peut dire que plusieurs décennies ont été complètement perdues pour bon nombre de villages du Sud. ${ }^{26}$

L'article était accompagné de cinq photographies que Patellani avait prises dans les salles de classe de l'école rurale de Jotta et de trois autres prises à Ghiandaro. ${ }^{27}$

Aujourd'hui, ces clichés ont le même impact visuel qu'à l'époque - et le même que les photographies d'Africo prises par Petrelli quatre ans plus tôt. Mais tandis que L'Europeo, comme nous l'avons vu, utilisait les prises de vue de Petrelli pour des raisons idéologiques pendant la campagne électorale houleuse de l'époque, Patellani utilisait son appareil photo pour enregistrer l'état pitoyable des écoles rurales du Sud (les deux villages calabrais servant d'exemples) non seulement pour attirer l'attention du public sur le problème, mais aussi pour dénoncer le régime fasciste, qui avait longtemps dissimulé l'état honteux des bâtiments scolaires du Sud et avait gaspillé les deniers publics à construire des bâtiments monumentaux dont le seul objectif était une autoglorification pompeuse. ${ }^{28}$ L'exclusion du Sud du débat public pendant plus d'une décennie à la suite des directives sur les médias formulées par l'attaché de presse de Benito Mussolini en $1931^{29}$ et la proclamation mensongère par le régime qu'il avait résolu la questione meridionale ont eu pour résultat que « des dizaines d'années avaient été complètement gaspillées » dans d'innombrables villages du Sud. Une fois de plus, les images décrivaient une société en retard sur son temps, et certainement pas cette modernité que le reste du pays en quête de

$\overline{26}$ «Si è fatto ben poco per i bambini che vanno a scuola in certe zone del Sud. È con un punto di rimorso che si pensa a tanti pomposi edifici pubblici delle grandi città (alle stazioni ferroviarie con i colonnati assiro-babilonesi, alle marmoree città-esposizioni, ed ai mausolei postelegrafonici), vedendo le aule di Jotta, di Ghiandaro, di Matera e degli altri innumerevoli paesi calabresi, lucani e pugliesi. Questi paesi dove i bambini crescono su banchi impossibili, ripetendo le classi fino a che giunge l'ora di andare a lavorare e di mettere la scuola tra i ricordi. Un ricordo di fili di paglia e di calcinacci che cadono dal soffitto. [...] Insomma questo viaggio nella regione delle scuole inverosimili mi ha condotto a concludere che in certe zone tutto è da fare. [...] Senza esagerare si può dire che in tante località del Sud sono andati completamente perduti parecchi decenni. » Ibid., p. 46-47. 27 Seulement trois de ces photographies sont reproduites dans cet article (fig. 3, 4 et 5). L'ensemble du reportage, qui se trouve maintenant au Museo di Fotografia Contemporanea (Musée de la photographie contemporaine) à Cinisello Balsamo, contenait plus de trente photographies.

28 Voir à ce sujet le travail de Bruno Tobia, en particulier Salve o popolo d'eroi... : la monumentale fasciste nelle fotografie dell'Istituto Luce. Rome : Editori Riuniti 2002.

29 Ces directives prévoyaient notamment de vérifier si les rapports et les articles étaient « utiles ou nuisibles à l'Italie et au régime » (point 2), d'éliminer les « informations alarmistes, pessimistes, catastrophiques ou déprimantes » (point 4) et d'éviter de donner « au reste du monde l'impression d'une misère profonde qui n'existe pas » (point 5). Voir Riccardo Cassero : Le veline del Duce : come il fascismo controllava la stampa. Milan : Sperling \& Kupfer Editori 2004, p. 10-11. 
rédemption, de seconde chance, était si occupé à poursuivre. Pourtant, alors même que Patellani publiait son exposé, conscient de l'impact émotionnel de ses photographies capables de décrire « ces scénarios incroyables mieux que tous les mots », il est intéressant qu'il mentionne aussi l'enquête menée simultanément par un journaliste britannique qui a vu et noté les mêmes impressions et qui discréditerait ainsi le système éducatif national italien aux yeux du monde. ${ }^{30}$ Il fallait donc que son désir d'exposer la situation surmonte sa modestie naturelle, qui aurait bien voulu protéger - contre toute évidence - l'image publique d'une institution telle que l'école, dont le rôle crucial dans la modernisation du pays était universellement reconnu.

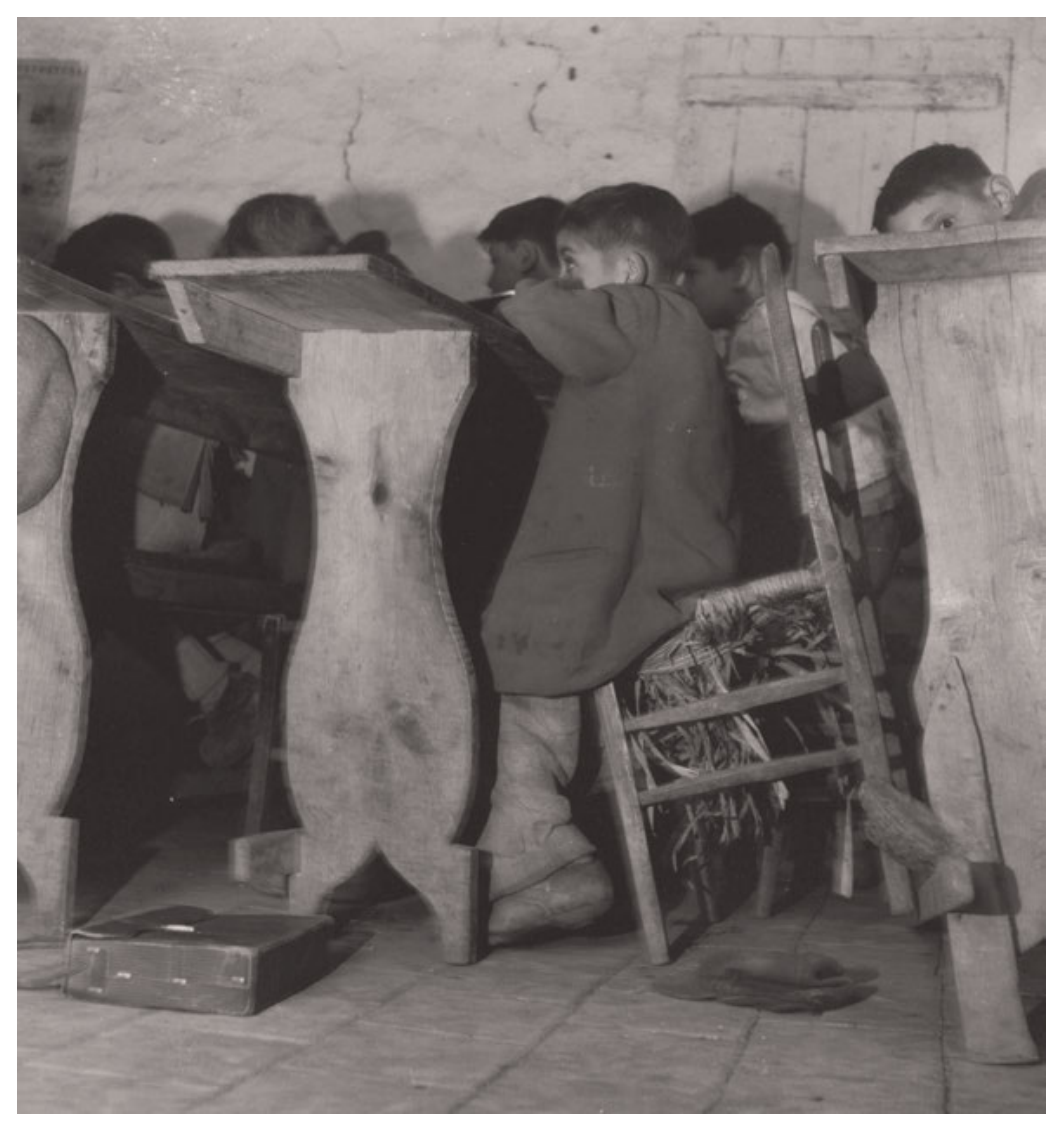

Fig. 3.5 Federico Patellani, Intérieur de l'école primaire de Jotta (1952) @ Studio Federico Patellani Région de Lombardie / Musée de la photographie contemporaine, Milan-Cinisello Balsamo.

30 Il n'a pas été possible d'identifier ce journaliste. Cependant, ce ne pouvait pas être David Seymour, puisqu'il a fait son reportage en 1950 . 


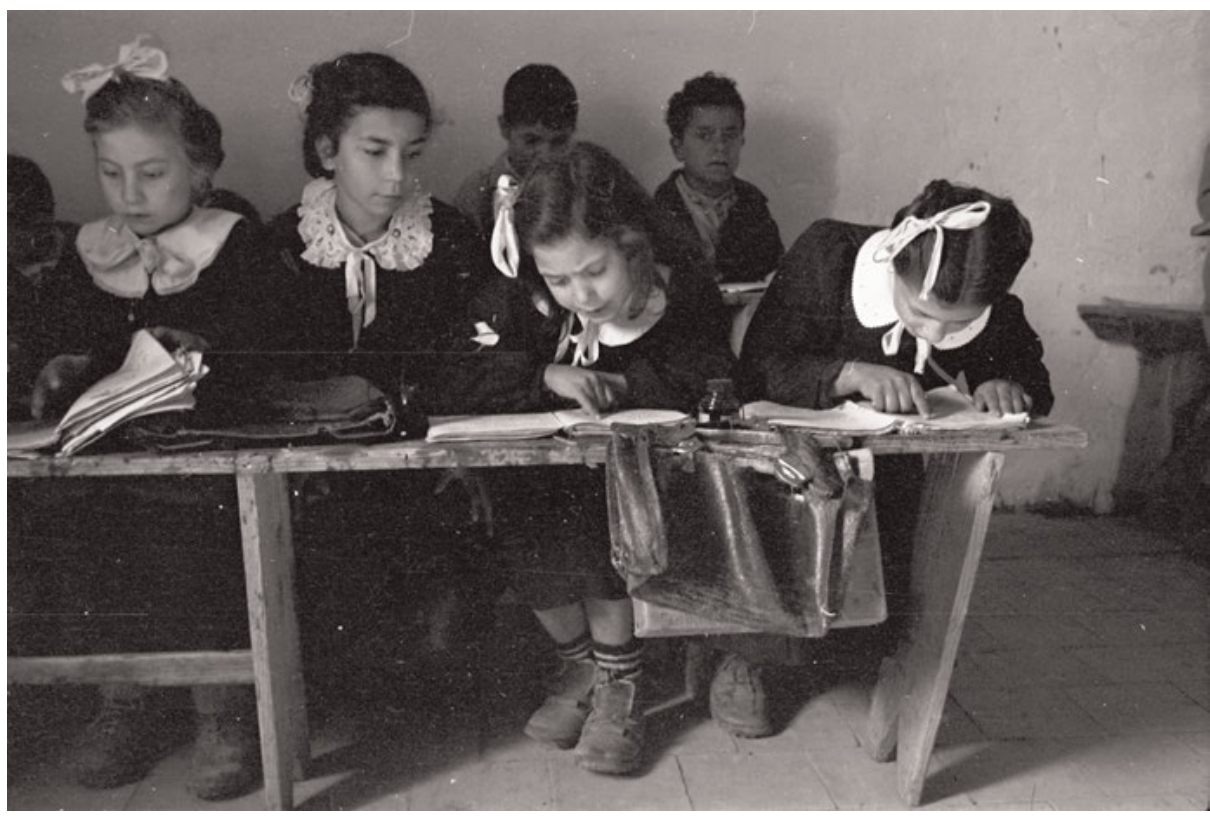

Fig. 3.6 Federico Patellani, Intérieur de l'école primaire de Ghiandaro (1952) (C) Studio Federico Patellani - Région de Lombardie / Musée de la photographie contemporaine, Milan-Cinisello Balsamo.

Les « écoles impossibles » de Jotta et de Ghiandaro ont provoqué une immense effervescence dans tout le pays et une nouvelle vague d'enquêtes sur l'état de l'éducation dans les régions méridionales. Il est intéressant de noter que même dans le cadre des enquêtes menées par des fonctionnaires du ministère et des pédagogues, on s'est mis à largement utiliser une documentation photographique. Nous pouvons en voir des exemples dans les articles publiés par Gustavo Sessa sur les bâtiments ruraux du Sud (avec une photo de l'école rurale de Vallazze, une banlieue de Cercemaggiore en Molise et une gravure du XIX ${ }^{\mathrm{e}}$ siècle d'Antonio Piccinni intitulée Scoletta rurale) ${ }^{31}$ et de Luigi Volpicelli sur « l'agonie de l'école » dans le Sud (avec deux photographies, l'une prise par Giovan Battista Poletto quelques années auparavant ; voir fig. 7). ${ }^{32}$ L'année suivante, l'hygiéniste Giuseppe Sangiorgi - adoptant la devise inventée par Patellani, lequel s'était inspiré d'une phrase prononcée par un enseig-

31 Gustavo Sessa : La casa della scuola. In : Il Mezzogiorno : rassegna della vita e dei problemi del Sud (mai 1952), p. 23-25.

32 Luigi Volpicelli : Il martirio della scuola nel Mezzogiorno. In : Il Mezzogiorno : rassegna della vita e dei problemi del Sud (novembre 1952), p. 12-14. 
nant du district de Matera $^{33}$ - publia dans une revue médicale réputée un article intitulé « Cristo si è fermato a Jotta » (Le Christ s'est arrêté à Jotta). ${ }^{34}$ Il y fait l'éloge de l'enquête parrainée par Epoca au motif qu'elle avait « touché les fibres les plus sensibles de notre cœur » et proposait que chaque Rotary club d'Italie « adopte une petite école rurale, à condition qu'elle soit aussi pauvre que l'école de Jotta $"{ }^{35}$ En 1954, l'article de Patellani a également été retenu comme témoignage visuel des conditions épouvantables des écoles rurales de la région, dans un rapport national de la Commission italienne pour l'étude des zones sous-développées au sein d'une section consacrée à la santé et à l'hygiène en Calabre. ${ }^{36}$ L'écho de l'agonie de l'école calabraise devait persister jusqu'en 1963, en plein milieu du prétendu « boom économique ", lorsque le magazine du Touring Club publia un article du journaliste Franco Abruzzo sur l'état pitoyable des écoles en Calabre, illustré par une série de photographies révélatrices de la FEMIA montrant des élèves de l'école primaire de San Nicola, dans la province de Reggio Calabria, assis pieds nus derrière de vieux bureaux bancals en bois (fig. 8). ${ }^{37}$

33 Patellani : Le scuole impossibili, p. 44. La citation exacte de l'instituteur était : « Per Carlo Levi Cristo si è fermato a Eboli. Ma per noi Cristo si è fermato a Matera. " (Selon Carlo Levi, le Christ s'est arrêté à Éboli. Mais, à notre avis, le Christ s'est arrêté à Matera.) Il se réfère ici au roman autobiographique de Carlo Levi, Cristo si è fermato a Eboli (Le Christ s'est arrêté à Éboli), un chef d'œuvre du néoréalisme italien publié par Einaudi en 1945.

34 Giuseppe Sangiorgi : Cristo si è fermato a Jotta. In : Athena : rassegna mensile di biologia, clinica e terapia $19, \mathrm{n}^{\circ} 12$ (1953), p. 393-395.

35 Ibid., p. 394. Cet article est basé sur un rapport délivré au Rotary Club de Bari le 24 mars 1953. 36 Francesco Serra/Mario Misasi : La Calabria. In : Centro nazionale di prevenzione e difesa sociale (éd.) : Atti del Congresso internazionale di studio sul problema delle aree arretrate (Milano, 10-15 ottobre 1954), vol.1: Rapporto della commissione italiana di studio sulle aree arretrate italiane. Milan : Giuffrè 1954, p. 891-979 ; voir surtout le passage intitulé « Condizioni culturali », p. 974-976. 37 Voir Franco Abruzzo : Calabria senza scuole. In : Le Vie d'Italia (avril 1963), p. 437-448. 


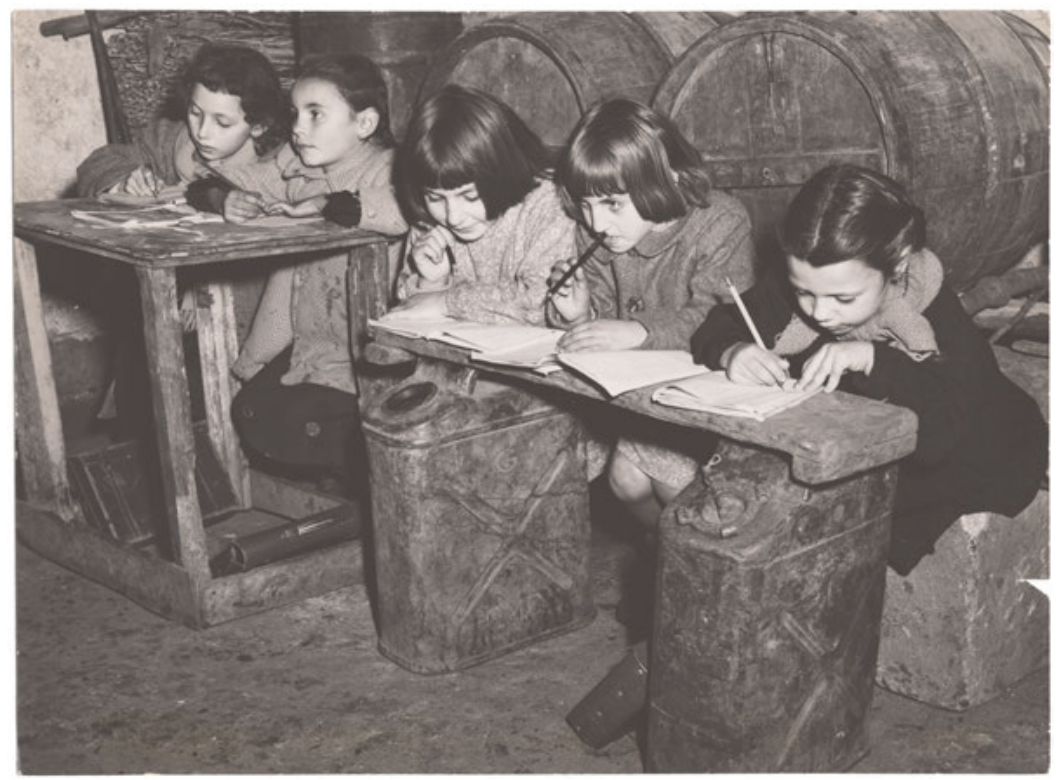

Fig. 3.7 Giovan Battista Poletto, Intérieur d'une école primaire dans une localité inconnue (1950 ?) (C) Archives municipales de Turin, fonds " Gazetta del Popolo », photothèque 1, dossier 77, fiche 5230.

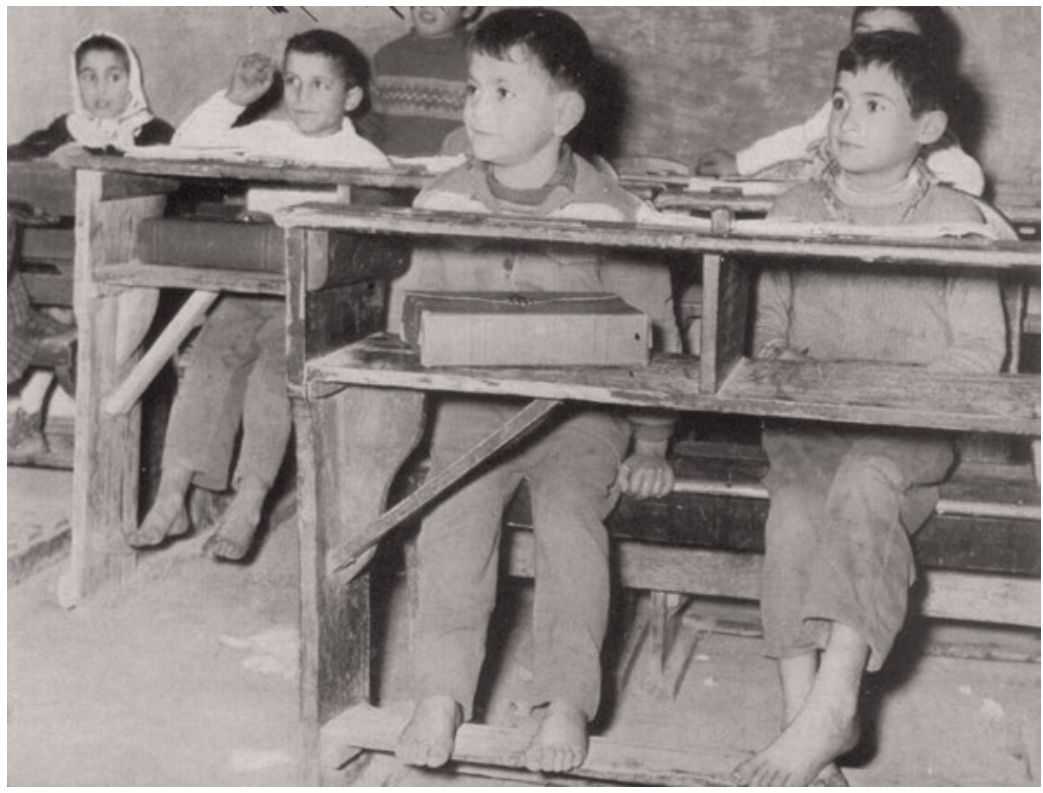

Fig. 3.8 Intérieur de l'école primaire de San Nicola (1963) @ reproduit de Le Vie d'Italia (avril 1963), p. 443. 


\section{Les photoreportages politiquement engagés d'Ando Gilardi, 1954-1957}

Nous avons vu comment le reportage photographique entrepris par Tino Petrelli en 1948 avait été utilisé à des fins de propagande par l'hebdomadaire à tendance libérale L'Europeo pour dénoncer les échecs du régime fasciste et pour condamner la tentative cynique des communistes d'exploiter la misère persistante des paysans du Sud dans le cadre de leurs attaques contre les politiques sociales et économiques du nouveau gouvernement démocrate-chrétien. De fait, l'historienne de la photographie Martina Caruso a récemment souligné comment, à cette époque, le reportage photographique était devenu un outil de propagande visuelle utilisé aussi bien par des magazines illustrés à tendance libérale tels que L'Europeo, Il Mondo, Epoca et Tempo, que par des magazines communistes tels que Politecnico, Vie Nuove, Noi Donne et Il Lavoro. ${ }^{38}$

C'est particulièrement vrai quand on considère que, dans l'après-guerre, le Parti communiste italien (Partito Comunista Italiano, PCI) qui historiquement avait eu sa base politique dans les régions les plus industrialisées du nord, avait ouvert une vaste enquête approfondie sur la questione meridionale en soutenant la campagne du mouvement paysan pour l'occupation et la redistribution des terres en friches appartenant aux grandes familles propriétaires. ${ }^{39}$ L'intellectuel le plus engagé de cette campagne a été Mario Alicata, secrétaire régional du Parti communiste calabrais. Il a également été membre du conseil d'administration du Comitato nazionale per la rinascita del Mezzogiorno (Comité national pour la relance du Sud), constitué par le PCI en 1947, qui a ouvert en 1950 une enquête sur les conditions de travail de la population méridionale, publié par la suite dans l'hebdomadaire communiste $L a$ Voce del Mezzogiorno. ${ }^{40}$ Dès lors, l'attention portée par la presse communiste aux conditions de vie morales et matérielles dans les régions méridionales, et en particulier au fléau de l'analphabétisme, grandit rapidement.

Ce n'est pas une simple coïncidence si les photographies de Petrelli - quatre ans après leur parution dans L'Europeo - ont été à nouveau utilisées par le mensuel communiste Noi Donne, qui a clairement indiqué qu'elles avaient déjà été publiées

38 Martina Caruso : Italian Humanist Photography from Fascism to the Cold War. Londres : Bloomsbury 2016, p. 94 ; voir en particulier le chapitre « Christ Stopped at Eboli : an Anthropology of the South » et le passage " The Politics of Miseria and the Communist Debate », p. 94-98.

39 Sur cette question en particulier, voir Sidney G. Tarrow : Peasant Communism in Southern Italy. Londres : Yale University Press 1967 ; Antonello Mattone : Partito comunista e contadini nel Mezzogiorno. In : Studi Storici 14, n ${ }^{\circ} 4$ (1973), p. 941-952 ; Vincenzo Mauro : Lotte dei contadini in Calabria: testimonianze sulle lotte dei braccianti negli anni 1944-1954. Milan : Sapere 1973.

40 La Voce del Mezzogiorno (15 janvier 1952) ; Mario Alicata : La cultura meridionale. In : Rinascita (octobre 1949), p. 438-442 ; Mario Alicata : Il meridionalismo non si può fermare ad Eboli. In : Cronache Meridionali (septembre 1954), p. 585-603. 
à des fins de propagande par un magazine bourgeois qui ne prenait même pas la peine de tenir compte de la réalité des écoles, des problèmes de l'analphabétisme ou du travail des mineurs d'âge, auxquels le Front populaire attribuait une telle importance. ${ }^{41}$ Le parti communiste a en fait rallié ses différents médias autour de cette question. Les portraits les plus efficaces des conditions de vie dans les écoles du Sud sont les reportages photo réalisés par le photographe Ando Gilardi pour le magazine hebdomadaire illustré de la Confédération italienne générale du travail (CGIL) ${ }^{42}$ Il Lavoro entre 1954 et $1956 .{ }^{43}$

Gilardi avait déjà dénoncé la situation des écoles dans une série d'articles intitulés « Inchiesta sulla donna italiana » (Enquête sur la femme italienne), publiés dans Il Lavoro en 1954. Dans le cadre de cette série, il écrivit un article déplorant le fait que les jeunes filles quittent prématurément l'école, illustré par une photographie de l'entrée d'une école de filles, installée dans une hutte en bois préfabriquée et délabrée dans la banlieue de Rome. Il écrivait :

Si nous pouvions pénétrer dans les maisons des élèves qui se sont égarés à partir de l'école primaire, nous verrions que ce sont toujours des filles de chômeurs et de paysans, et en général de travailleurs pauvres. Année après année, les filles d'ouvriers et d'employés, et même celles de nombreux artisans et professionnels, abandonnent leurs études. À quelques rares exceptions près, seuls les membres des classes supérieures échappent à cette sélection cruelle, qui se fait exclusivement sur la base de considérations économiques. ${ }^{44}$

41 Bambini italiani 1952. In : Noi Donne (20 avril 1952), p. 6-7.

42 Le CGIL était un syndicat national de gauche, fortement influencé par le Parti communiste.

43 Ando Gilardi (1921-2012) a commencé sa carrière de photographe professionnel en 1945. Il a été journaliste et photoreporter d'abord pour le journal L'Unità et ensuite pour les magazines Il Lavoro et Vie Nuove, tous publiés par le Parti communiste italien. Durant les années cinquante et soixante, il a collaboré avec les anthropologues Ernesto de Martino et Diego Carpitella en réalisant des photoreportages ethnographiques sur les traditions populaires dans les régions du Sud. Pendant les années qui ont suivi, il a concentré son énergie à la recherche photographique et a publié divers livres, notamment Storia sociale della fotografia. Milan : Feltrinelli 1976. En 1979, il a fondé le groupe Foto/ gram qui rassemblait des professeurs et des étudiants, et qui a lancé des cours novateurs sur l'usage de la photographie dans le cadre de l'éducation dans les écoles italiennes. Pour plus d'information, voir Ando Gilardi, Olive e bulloni : lavoro contadino e operaio nell'Italia del dopoguerra, 1950-1962, édité par Fabrizio Urettini. Milan : Edizioni Fototeca Storica Nazionale 2009; Daniela Giordi (éd.) : Ando Gilardi reporter: Italia 1950-1962. Cinisello Balsamo : Silvana 2019.

44 "Se noi potessimo entrare nella casa delle scolare che si sono perse per via, dalla prima elementare in su, vedremo che di volta in volta trattasi di figlie di disoccupati, di contadini e in genere di lavoratori poverissimi e poveri. Abbandonano poi gli studi, di anno in anno, le figlie degli operai e degli impiegati e persino quelle di molte categorie artigianali e professionali. Tranne rarissime eccezioni, da questa crudele selezione che ubbidisce esclusivamente a un principio economico, si salvano solo le appartenenti alle categorie agiate e ricche. » Ando Gilardi : Soltanto una su cento. In : Il Lavoro (21 février 1954), p. 2-3. 
Gilardi avait déjà abordé les problèmes de l'école et de l'enfance en tant que correspondant calabrais d'Il Lavoro. En fait, il avait été chargé de faire un reportage sur la ville calabraise de Melissa, sur le plateau de Sila, où, le 29 octobre 1949, la police avait ouvert le feu sur une foule de gens pauvres et sans terres qui avaient occupé de grands domaines agricoles, tuant trois jeunes et en blessant quinze.

Le massacre de Melissa, qui suivait celui de Portella della Ginestra le $1^{\text {er }}$ mai 1947 et annonçait celui de Torremaggiore le 29 novembre 1949, avait eu des répercussions nationales, provoquant une vague d'indignation dans tout le pays. Par conséquent, le Parti communiste et la CGIL investirent beaucoup de ressources pour obtenir des informations précises sur ce qui s'était passé, afin de mettre en lumière les responsabilités de la police et du gouvernement. En 1949, le réalisateur Carlo Lizzani dirigea l'enquête cinématographique Nel Mezzogiorno qualcosa è cambiato (Quelque chose a changé dans le Sud), écrite par Mario Alicata et produite par la société de production cinématographique Libertas Film. Documentant les efforts considérables déployés par les mouvements ouvriers et paysans pour le développement social et économique des régions du sud et le travail réalisé par l'Assise per la rinascita del Mezzogiorno (Assemblée pour le renouveau de l'Italie méridionale), le film s'achève sur des images d'une manifestation politique à Melissa. ${ }^{45}$

Cet engagement du Parti communiste et des syndicats de gauche s'est poursuivi au cours des années suivantes. Durant l'été 1954, Il Lavoro décida de commémorer la mort, cinq ans plus tôt, de trois jeunes manifestants et de dénoncer la misère économique dans laquelle la population rurale du Sud vivait toujours. Pendant son séjour en Calabre, Gilardi a également pu documenter les piètres conditions sanitaires du bidonville surnommé "Shangai » à Crotone, le principal centre industriel calabrais de la côte ionienne, dont les portraits d'enfants pieds nus et crasseux évoquaient ceux de Petrelli à l'école d'Africo. Gilardi a pris quelques photos de la baraque délabrée faite de planches en bois et de tôles métalliques servant de garderie pour les enfants après l'école dans le village où vivent les familles des travailleurs des usines de produits chimiques de Montecatini. ${ }^{46}$ Il se rendit ensuite à l'intérieur des terres, à Melissa, et commença à préparer son rapport qui devait être publié dans le numéro du 31 octobre 1954.

À l'intérieur de la petite école primaire de la ville, Gilardi a pris de nombreuses photos représentant des garçons et des filles d'âges différents, assis à leur bureau en train d'assister aux leçons de leur instituteur. Ces photographies, conservées aujourd'hui dans les archives photographiques de Gilardi, n'ont toutefois jamais été

45 Ce film documentaire est conservé dans le fonds audiovisuel du Parti communiste italien aux Archivio audiovisivo del movimento operaio e democratico (Archives audiovisuelles du mouvement ouvrier et démocratique) à Rome.

46 Les photos prises à Crotone n’ont pas été publiées dans Il Lavoro, mais sont conservées dans la Fototeca Storica Nazionale Ando Gilardi (Photothèque historique nationale Ando Gilardi) à Milan. 
publiées. ${ }^{47}$ L'article d'Il Lavoro était accompagné d'une seule photo, devenue très célèbre, représentant une rangée d'écoliers assis sur un banc accueillant le photographe le poing levé (fig. 9). La légende de cette photo était libellée comme suit : « Leurs parents leur ont enseigné ce salut lorsque l'INCOM est venu pour leur éviter la honte de prêter leur image à la propagande des classes dirigeantes. Ils le répètent toujours devant tout objectif. ${ }^{48} C^{\prime}$ est Gilardi lui-même qui a expliqué plus en détail les raisons de ce salut :

Après le massacre, au moment de l'occupation des terres en friche, les cameramen de la Settimana INCOM se sont rendus à Melissa pour tourner un film de propagande. Les gens se sont réunis pour discuter du meilleur moyen pour éviter d'être exploités, même de loin, par la propagande gouvernementale. Divers moyens ont été suggérés, certains impétueux ; ils ont finalement trouvé une solution pacifique : chaque fois qu'une personne était photographiée, elle devait immédiatement saluer en levant un poing fermé. Les mères ont même appris ce geste de révolte à leurs enfants, et personne ne l'a oublié. Même de nos jours à Melissa, il suffit de braquer l'appareil pour provoquer la levée d'une foule de poings serrés. Pour avoir des photos normales, il faut y aller y en compagnie d'un habitant du coin : "C'est un camarade », expliquera votre guide local aux enfants, qui retourneront immédiatement à leurs jeux ou poseront normalement. ${ }^{49}$

47 Dix de ces photos ont été retrouvées dans la Fototeca Storica Nazionale Ando Gilardi à Milan. 48 « Gli insegnarono questo saluto quando venne la INCOM : per salvarli dall'onta di prestare la loro immagine alla propaganda dei padroni. Lo ripetono immancabilmente davanti a qualsiasi obbiettivo. » Ando Gilardi : Melissa 1954. In : Il Lavoro (31 octobre 1954), p. 7-9.

49 «Una volta, dopo l'eccidio, al tempo della distribuzione delle terre, salirono a Melissa gli operatori della Settimana INCOM per girare un documentario di propaganda. La gente si riunì per discutere il modo migliore per evitare di servire, anche indirettamente, alla propaganda governativa. Vari modi, alcuni spicciativi, vennero suggeriti, poi trovarono una soluzione pacifica : ogni qualvolta la macchina da presa era diretta verso qualcuno, subito egli alzava il braccio salutando a pugno chiuso. Anche ai bambini le madri insegnarono il gesto ribelle, e nessuno più lo ha dimenticato, e a Melissa ancor oggi basta puntare la macchina fotografica per provocare il saluto a pugno chiuso. Per avere fotografie normali, bisogna andare in giro accompagnati da qualcuno : ‘ C'è un compagno > spiega l'accompagnatore ai bambini e allora essi ritornano ai loro giochi, o si mettono in posa normalmente. » Gilardi : Melissa 1954, p. 8. La Settimana INCOM était un court-métrage d'actualités italien, distribué chaque semaine dans les cinémas de 1946 à 1965 par la société de production cinématographique INCOM (Industria Corti Metraggi Milano). Il n'est pas clair à quel film d'actualités Gilardi fait référence. Dans les archives de l'Institut LUCE qui ont acquis la marque INCOM en 1967 et conservent leurs archives, on a retrouvé un court métrage intitulé Minister Segni $a$ Melissa (5'), qui n'indique ni la société de production ni la date de tournage (code : D063206). De plus, les archives incluent également l'épisode $n^{\circ} 412$ de la Settimana INCOM du 8 mars 1950, dirigé par Stefano Canzio, et entièrement consacré à la Calabre, toujours sans aucune référence directe à Melissa. Une scène remarquable du film montre plusieurs étudiants qui vont à l'école en suivant une route de campagne, avec cette voix off : "Nous extirperons également toutes les mauvaises herbes de l'analphabétisme : la route est ouverte, nous devons l'améliorer » (13.00'-13.03'). 


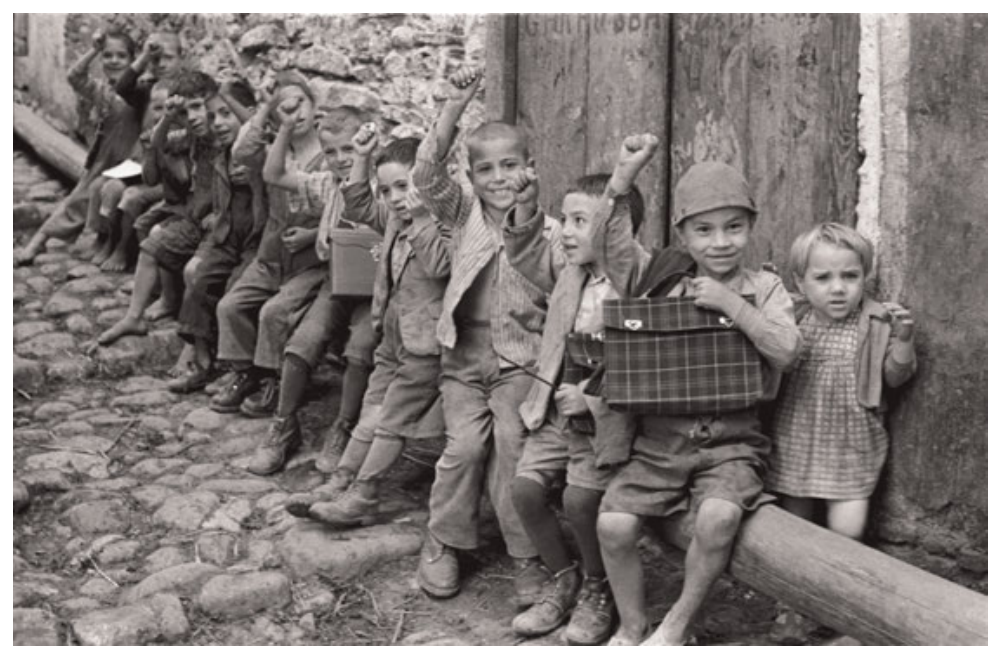

Fig. 3.9 Ando Gilardi, Les écoliers de Melissa saluent en levant le poing (1954) @ Fototeca Storica Nazionale Ando Gilardi, Milano.

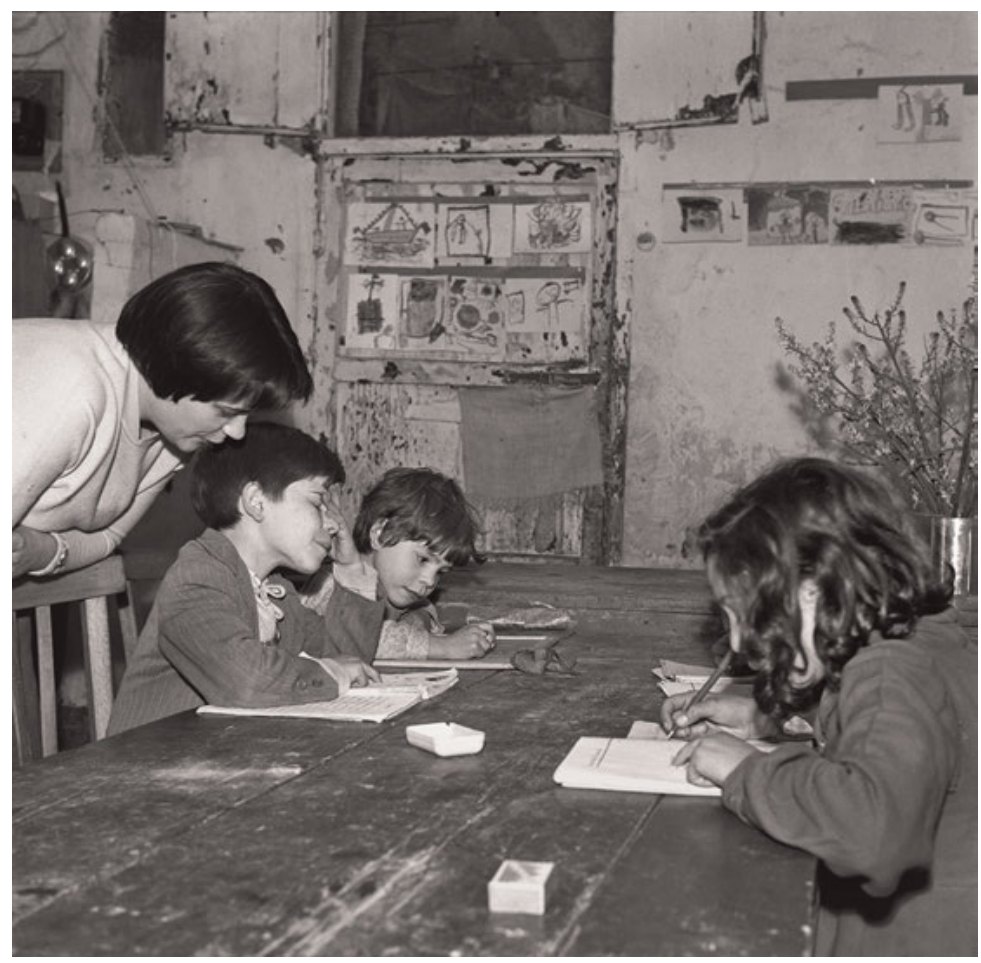

Fig. 3.10 Ando Gilardi, La garderie Goffredo Fofi à Palerme (1956) (c) Fototeca Storica Nazionale Ando Gilardi, Milano. 
$\mathrm{Au}$ cours des années suivantes, les enfants de Melissa, cartables sous les bras et poings levés, devinrent l'emblème du mouvement paysan du Sud qui avait subi une répression féroce de la part de la police dans cette ville très calabraise. Ando Gilardi reviendrait quelques années plus tard pour photographier des écoliers dans le cadre de l'enquête journalistique "Indagine nel Mezzogiorno " (Enquête dans le Sud). Publiée une fois encore dans l'hebdomadaire illustré de la CGIL, elle l'a amené à visiter la garderie après l'école pour les enfants pauvres fondée par le professeur Goffredo Fofi dans le quartier de Cortile Scalilla à Palerme. ${ }^{50}$ En 1955, Fofi avait été licencié de Partinico pour avoir traité le problème de l'éducation populaire sans autorisation préfectorale, ${ }^{51}$ et bien que sans revenu mais avec le soutien de Lucio Lombardo Radice ${ }^{52}$ - qui lui avait consacré un éditorial dans l'organe officiel du PCI, L'Unità ${ }^{53}$ - Fofi a fondé sa garderie après l'école à Palerme. Gilardi a pris de nombreuses photos de salles de classe improvisées remplies d'enfants sales vêtus de lambeaux et ses images ont fourni un témoignage d'une force extraordinaire de la lutte contre l'analphabétisme menée par un seul enseignant communiste disposant de peu de moyens. ${ }^{54}$ Une seule de ces photos, pourtant, sera publiée dans le troisième volet de l'" Indagine nel Mezzogiorno » : elle représente un enfant qui lit péniblement avec l'aide de l'épouse de Fofi, Michela, tandis que deux autres enfants, assis à la même grande table, font leurs devoirs (fig. 10). ${ }^{55}$ Cette photo représentait une métaphore efficace des terribles difficultés rencontrées par les enfants pauvres et ouvriers pour se libérer de l'ignorance séculaire dans laquelle on avait maintenu les classes populaires, et décrivait les premières pratiques d'éducation démocratique et coopérative qui se répandaient dans toute l'Italie dans le cadre du développement du Mouvement des écoles modernes.

50 En 1955, Goffredo Fofi (né en 1937) s'est rendu à Partinico, où Danilo Dolci prêchait la lutte nonviolente contre la mafia et pour les droits des travailleurs au moyen des scioperi a rovescio (littéralement « manifestations à l'envers »); au cours des années suivantes, Fofi poursuivit son engagement en faveur de l'éducation populaire dans les régions les plus défavorisées d'Italie du sud.

51 Les préfectures - connues de nos jours également sous le nom de bureaux territoriaux gouvernementaux - sont les agences du ministère de l'Intérieur qui représentent le gouvernement central dans chaque province.

52 Lucio Lombardo Radice (1916-1982), fils de Giuseppe Lombardo Radice (un éducateur qui, depuis les années 20, avait introduit en Italie les principes du Mouvement moderne de l'école), hérita de l'intérêt de son père pour les problèmes de l'école et fonda la revue Riforma della Scuola, qui devint rapidement l'organe dirigeant de l'éducation progressiste. Il était aussi un dirigeant du Parti communiste italien.

53 L. Lombardo Radice : Reato d'alfabeto. In : L’Unità (8 février 1956), p. 2. L'article a été reproduit également dans Adolfo Battaglia (éd.) : Dibattito sulla scuola. Bari : Laterza 1956, p. 290.

54 Quinze de ces photos ont été trouvées à la Fototeca Storica Nazionale Ando Gilardi à Milan.

55 Ando Gilardi : A te : ti affitti ? In : Il Lavoro (14 avril 1957), p. 4-5. 